medgen $2018 \cdot 30: 259-266$

https://doi.org/10.1007/s11825-018-0193-3

Online publiziert: 10. Juli 2018

(c) Der/die Autor(en) 2018

CrossMark

\author{
Thomas Bajaj' · Alfredo Ramirez ${ }^{1,2} \cdot$ Holger Wagner-Thelen' \\ 'Sektion für Neurogenetik und Molekulare Neuropsychiatrie an der Klinik für Psychiatrie und \\ Psychotherapie, Uniklinik Köln, Köln, Deutschland \\ ${ }^{2}$ Klinik für Neurodegenerative Erkrankungen und Gerontopsychiatrie, Universitätsklinikum Bonn, Bonn, \\ Deutschland
}

\title{
Genetik der Alzheimer-Krankheit
}

\section{Entschlüsselung von pathologisch veränderten zellulären Systemnetzwerken}

Zahlreiche neurodegenerative Erkrankungen, so auch die Alzheimer-Erkrankung („Alzheimer's disease“, AD), sind von multifaktorieller Natur. Neben exogenen, umwelt- und lebensstilbedingten Faktoren nehmen insbesondere genetische und epigenetische Faktoren Einfluss auf die Pathogenese der AD [62]. Seit über 30 Jahren trägt die Genforschung bereits systematisch dazu bei, die genetische Architektur der AD besser zu verstehen. Das Vorhaben der Wissenschaftler ist es, die der $\mathrm{AD}$ zugrunde liegenden pathophysiologischen $\mathrm{Me}$ chanismen durch die Erforschung der genetischen Ursachen zu entschlüsseln.

\section{Formen der Alzheimer- Krankheit}

In den 1990er-Jahren konnten die für die monogenen, familiären, frühmanifesten Formen der AD („early-onset“ AD [EOAD], Ersterkrankungsalter $<65$ Jahre, Prävalenz 1-5\%) verantwortlichen Gene auf den Chromosomen 21q21.2 [59], 14q24.3 [61] und 1q42.13 [37] identifiziert werden. Diese kodieren jeweils für das Amyloid-Vorläuferprotein („amyloid- $\beta$ precursor protein“, APP), Presenilin 1 (PSEN1) und dessen Homolog Presenilin 2 (PSEN2). Obgleich hochpenetrante Mutationen in den o.g. Genen äußerst selten sind (5-10\% der EOAD-Fälle) [5], gewährte die funktionelle Charakterisierung dieser aufschlussreiche Einblicke in die molekularen Pathomechanismen, insbesondere der $\beta$-Amyloidogenese, und erweiterte gleichermaßen das Verständnis für die wesentlich häufiger auftretende, oft sporadische, spätmanifeste Form der $\mathrm{AD}$ („late-onset“ $\mathrm{AD}$ [LOAD], Ersterkrankungsalter $>65$ Jahre, Prävalenz $>95 \%$ ).

\section{Bedeutung der Genetik bei der LOAD}

Im Gegensatz zur EOAD lassen sich bei der LOAD keine eindeutigen, dominanten Übertragungsmuster feststellen, da diese nicht auf pathogene Mutationen in APP, PSEN1 oder PSEN2 zurückzuführen ist [62]. Obgleich bei der LOAD eine familiäre Häufung zu verzeichnen ist, stellen Familien mit mehreren erkrankten Angehörigen eine Ausnahme dar. Daher sind Assoziationsstudien für die Identifizierung genetischer Risikofaktoren unabdingbar. Anfang der 1990er-Jahre gelang es, eine Assoziation zwischen dem APOE \&4-Allel aufChromosom 19q13.32 und dem Auftreten einer AD nachzuweisen und diese in den folgenden Jahren aufgrund der hohen Prävalenz von APOE $\varepsilon 4$ bei AD-Betroffenen konsistent zu replizieren. Bis heute gilt das Vorliegen von ein oder zwei Kopien des APOE $\varepsilon 4$-Allels als stärkster Risikofaktor; nicht nur für die LOAD, sondern auch für die EOAD [2, 54, 63]. Das humane Apolipoprotein $\mathrm{E}$ (ApoE) existiert in den drei Isoformen ApoE2, ApoE3 und ApoE4, welche die Genprodukte von drei Allelen an zwei Einzelnukleotidpolymorphismen („single-nucleotide polymorphism“, SNP), rs429358 und rs7412, sind und sich hinsichtlich ihrer Aminosäuren an den Positionen 112 und 158 unterscheiden (ApoE2: C112/C158, ApoE3: C112/R158, ApoE4: R112/R158) [39]. Während die Präsenz eines APOE \&4-Allels das AD-
Risiko im Vergleich zum $\varepsilon$ 3-Allel bereits um das Dreifache steigert, erhöht es sich bei $\varepsilon 4 / \varepsilon 4$-Homozygotie auf das 12 - bis 15-Fache [2]. Hinzukommend existiert ein Gendosiseffekt, der bei $\varepsilon 4 / \varepsilon 4$-Homozygotie ein früheres Manifestationsalter der AD begünstigt [8]. Verschiedenartige Mechanismen sind für die Verknüpfung von ApoE4 und $\mathrm{AD}$ verantwortlich. Durch eine direkte Interaktion mit dem $\beta$-Amyloid-Peptid ( $A \beta$ ) vermag ApoE4 den Abbau von $A \beta$ zu modulieren [27]. Glial sezerniertes ApoE4 wirkt zudem stimulierend auf die neuronale $A \beta$ Produktion [22]. Weiterhin beeinflusst ApoE4 den Cholesterinmetabolismus in ungünstiger Weise [11] und spielt somit eine prädisponierende Rolle für die Entwicklung von kardiovaskulären Erkrankungen, die ihrerseits wiederum das Erkrankungsrisiko für die AD erhöhen [60]. Bemerkenswerterweise konnte für Träger des APOE $\varepsilon 2$-Allels eine reduzierte Suszeptibilität für die AD festgestellt werden, die womöglich auf neuroprotektiven Eigenschaften von ApoE2 beruht [7]. Das APOE \&3-Allel gilt als risikoneutral. Wie genau ApoE-Isoformen AD-prädisponierend oder $\mathrm{AD}$-protektiv wirken, ist unvollständig erfasst und bedarf weiterer Untersuchungen [27]. Obwohl APOE $\varepsilon 4$ den stärksten genetischen Risikofaktor für die $\mathrm{AD}$ darstellt, ist er nicht krankheitsverursachend und macht lediglich $27,3 \%$ der geschätzten Heritabilität von $58-79 \%$ aus [62]. Dementsprechend ist ein Großteil der Heritabilität ungeklärt [52]. 


\section{Genomweite Assoziationsstudi- en und Sequenziermethoden der nächsten Generation}

Um Polymorphismen zu identifizieren und die fehlende Heritabilität für die LOAD zu erklären, hat sich ein populäres Studiendesign in der genetischen Epidemiologie bewährt. Der Einsatz von hypothesenfreien, genomweiten Assoziationsstudien (GWAS) erlaubt einen möglichen Nachweis einer Genotyp-Phänotyp-Beziehung, der durch den punktuellen Vergleich der DNA-Sequenz an zahlreichen Loci in einer großen Anzahl erkrankter und nicht erkrankter Individuen erfolgt [31]. Die Anwendung metaanalytischer Methoden erhöht zudem die Aussagekraft von erzielten Ergebnissen und verringert gleichzeitig die Wahrscheinlichkeit von falsch-positiven Befunden. Aufgrund des Kopplungsungleichgewichtes (,linkage disequilibrium“, LD) zwischen benachbarten Varianten handelt es sich methodisch bedingt bei den identifizierten Varianten in den seltensten Fällen um die kausal assoziierte Variante. Daher sind im Anschluss weitere Studien notwendig, die eine Feinkartierung der im LD befindlichen Varianten aufzeigen, um so die pathogene oder die pathogenen Varianten zu identifizieren. Im Rahmen von zwei groß angelegten GWAS im Jahr 2009 wurde nicht nur der genetische Risikofaktor $A P O E$ als solcher repliziert; insbesondere konnte die Anzahl von LOAD-assoziierten Suszeptibilitätsloci um drei weitere Risikogene erweitert werden [18, 33]. Zahlreiche GWAS und Sequenzierstudien, die in den folgenden Jahren durchgeführt wurden, trugen zur Aufdeckung von weiteren, häufiger repräsentierten und selteneren Suszeptibilitätslocibei [17, 21, 25, 26, 44,53].Im Jahr 2013 führte eine mehrstufige Metaanalyse im Rahmen des „International Genomics of Alzheimer's Project"(IGAP)Konsortiums unter Einsatz von 74.046 Proben zur Bestätigung von Ergebnissen vorangegangener GWAS und zur Detektion von elf neuen genomweit signifikanten Suszeptibilitätsloci für die LOAD [34]. Erst in jüngster Vergangenheit gelang es, drei weitere, seltene Genvarianten zu identifizieren [56]. Durch den technischen Fortschritt und den zunehmen- den Einsatz von GWAS und automatisierter Sequenziermethoden der nächsten Generation (,whole-exome/-genome sequencing", WES/WGS) gelingt es Wissenschaftlern sukzessive AD-Risiko-Loci zu identifizieren. Auf Basis der identifizierten Genvarianten erfolgt unter Einbeziehung von Signalwegen und den assoziierten Proteinen die detaillierte Entschlüsselung von pathologisch veränderten zellulären Systemnetzwerken. An der LOAD beteiligte zelluläre Funktionsmodule, welche durch Gen-Ontologie- und Signalweganalysen in den Vordergrund rücken, lassen sich mit Lipoproteinpartikeln, Cholesterin-Efflux, der Regulation von endozytotischen Prozessen und vor allem mit einer Immunantwort assoziieren [24]. Bemerkenswerterweise beobachten Wissenschaftler eine Kumulation von häufigen und seltenen Genvarianten, die sich diesen Funktionsmodulen zuordnen lassen [56]. In den nächsten Abschnitten erfolgt eine detaillierte Beschreibung ausgewählter Kandidatengene, die mit immunsystembezogenen Prozessen der AD in Verbindung gebracht werden können.

\section{Mikroglia vermittelte Immunantwort bei der LOAD}

Wichtige Erkenntnisse aus GWAS und Sequenzierstudien suggerieren, dass Mikroglia, die residenten Immunzellen des ZNS, eine entscheidende Rolle bei der Pathogenese der AD spielen. Eine beachtliche Anzahl der in genetischen Studien identifizierten Risikogene weisen immunsystembezogene Funktionen auf: CR1 („,complement receptor type 1“), CLU („,clusterin“) [18, 33], SPI1 („spi-1 protooncogene"), CD33 („,cluster of differentiation 33“), MS4A6A/MS4A6E („membrane spanning 4-domains $\mathrm{A} 6 \mathrm{~A} / \mathrm{A} 6 \mathrm{E}^{\text {") }}$, ABCA7 („ATP-binding cassette sub-family A member 7“), EPHA1 („ephrin receptor A1“), CD2AP („CD2-associated protein“) [21, 44], TREM2 („triggering receptor expressed on myeloid cells $2^{\text {") }}$ $[17,26]$, TYROBP (,TYRO protein tyrosine kinase-binding protein") [48], HLA-DRB5/DRB1 (,major histocompatibility complex, class II, DR $\beta 5 / 1^{\text {“) }}$, INPP5D (,inositol polyphosphate-5- phosphatase D“) [34], PLCG2 („phospholipase $\mathrm{C} \gamma 2$ ") und $A B I 3$ („B3-domaincontaining transcription factor $\mathrm{ABI} 3^{\text {") }}$ ) [56]. Hinzukommend werden zahlreiche dieser Gene in höchstem Maße von Mikroglia exprimiert [68].

\section{CR1}

CR1 auf Chromosom 1q32 kodiert für den Komplementrezeptor 1 , welcher die Faktoren $\mathrm{C} 3 \mathrm{~b}$ und $\mathrm{C} 4 \mathrm{~b}$ bindet und an der Regulation der Komplementaktivierung beteiligt ist [20]. CR1 zog insbesondere die Aufmerksamkeit von Alzheimer-Forschern auf sich, als Genvarianten im CR1-Locus identifiziert wurden, die mit einem erhöhten Risiko für die LOAD assoziiert sind. Der SNP rs6656401 zeigte dabei eine prominente Assoziation (,odds ratio“, $\mathrm{OR}=1,21$; $p=3,7 \times 10^{-9}$ ) [33]. Weitere SNPs im CR1-Locus, die mit dem LOAD-Risiko in Verbindung gebracht werden, sind rs1408077 (OR=1,17; $\left.p=8,3 \times 10^{-6}\right)$, rs6701713 (OR=1,17; $\left.p=8,7 \times 10^{-6}\right)$ und $\mathrm{rs} 3818361\left(\mathrm{OR}=1,17 ; p=9,2 \times 10^{-6}\right)$ [18]. Es ist wichtig zu erwähnen, dass es sich bei den o.g. SNPs nicht um unabhängige Assoziationssignale handelt, da sich die identifizierten Genvarianten im LD zueinander befinden. Aus diesem Grund sind weitere genetische und funktionelle Studien notwendig, um die tatsächliche(n) krankheitsbedingende(n) Genvariante(n) zu identifizieren und mögliche Pathomechanismen zu entschlüsseln. Auf funktioneller Ebene konnte bei Trägern des Risikoallels der SNPs rs670173 und rs3818361 bereits eine höhere CR1-Expression im Hirngewebe nachgewiesen und mit dem Krankheitsstatus korreliert werden [28]. Zudem ist der SNP rs6656401 mit einer erhöhten $A \beta$-Plaquebelastung assoziiert [6]. Weiterhin konnte eine positive Korrelation von vier SNPs (rs646817, rs1746659, rs11803956 und rs12034383) im CR1-Locus mit einer erhöhten Menge an $A \beta_{42}$ in der Cerebrospinalflüssigkeit festgestellt werden. Aufgrund des hohen LD der SNPs im CR1-Gen lässt sich nicht sagen, welcher/welche SNP(s) im LD-Block für die Erkrankung von funktioneller Bedeutung ist und zur Entstehung des untersuchten Phänotyps 
beiträgt. Zudem existiert eine Kopienzahlvariation im CR1-Locus, welche in einer Produktion verschiedener CR1Isoformen resultiert. Die längere, sogenannte CR1-S Isoform, ist mit der LOAD assoziiert, welches ein weiteres Indiz für die Beteiligung von CR1 an der Krankheitsentstehung ist [4].

\section{CD33}

Bei CD33 handelt es sich um einen Immunrezeptor, der zur Immunglobulin-Superfamilie zählt und dessen Gen auf Chromosom 19q13.3 lokalisiert ist [49]. Es konnte ein Risikoallel des SNP rs3865444, welcher genomweit mit der LOAD-Suszeptibilität assoziiert ist, identifiziert werden [21, 44]. Das C-Allel des SNP rs3865444 zeigt dabei die stärkste Risiko-Assoziation mit der LOAD, wohingegen das A-Allel protektiv zu sein scheint [44]. In funktionellen Untersuchungen konnte demonstriert werden, dass Träger des Risikoallels eine höhere CD33-Zelloberflächenexpression aufweisen. Darüber hinaus wurden eine verringerte Internalisierung von $A \beta_{42}$, eine erhöhte $A \beta$-Plaquebelastung und eine Zunahme an aktivierten Mikroglia beschrieben [3]. Interessanterweise wurde eine erhöhte Expression von CD33 im Gehirn mit einer verstärkten Abnahme der kognitiven Leistungsfähigkeit und dem klinischen Bild der AD in Zusammenhang gebracht [28]. Auf funktioneller Ebene wurde gezeigt, dass der SNP rs3865444 die Exon 2-Spleißeffizienz von CD33 moduliert [40]. In der vom IGAP-Konsortium im Jahr 2013 durchgeführten Metaanalyse lag der SNP rs3865444 knapp unter der genomweit signifikanten Grenze. Aus diesem Grund sind weitere Studien notwendig, um eine tatsächliche Assoziation von CD33 mit der LOAD zu bestätigen [34].

\section{TREM2}

Weitere Hinweise für eine Beteiligung von Mikroglia an der Pathogenese der $\mathrm{AD}$ rühren von der Entdeckung einer seltenen Genvariante in TREM2 her. Der SNP rs75932628 (p.R47H) erhöht das LOAD-Risiko um das Drei- bis Vierfache und kommt somit dem Asso-

medgen 2018·30:259-266 https://doi.org/10.1007/s11825-018-0193-3

(c) Der/die Autor(en) 2018

T. Bajaj · A. Ramirez · H. Wagner-Thelen

\section{Genetik der Alzheimer-Krankheit}

\section{Zusammenfassung}

Die Alzheimer-Erkrankung („,Alzheimer's disease", AD) ist die häufigste Ursache der neurodegenerativen Demenzen. Im Gegensatz zu monogenen und meist frühmanifesten Formen der $A D$, welche auf hochpenetrante Mutationen in den Genen $A P P, P S E N 1$ und PSEN2 zurückzuführen sind, wird die Suszeptibilität für die sporadische, oft spätmanifeste Form der AD durch eine komplexe Wechselwirkung zwischen genetischen und epigenetischen Faktoren wie auch umwelt- und lebensstilbedingten Faktoren bestimmt. Obgleich APOE \&4 der stärkste genetische Risikofaktor für die AD ist, macht der Effekt des APOE \&4 lediglich 27,3\% der geschätzten Heritabilität von 58-79\% aus. Durch den kontinuierlichen technischen Fortschritt von GWAS (genomweite Assoziationsstudien) und automatisierten Sequenziermethoden der nächsten Generation gelingt es Wissenschaftlern in groß angelegten Kollaborationen sukzessive die fehlende Heritabilität aufzudecken. Wichtige Erkenntnisse aus GWAS und Signalweganalysen suggerieren, dass Mikroglia, die residenten Immunzellen des ZNS, eine entscheidende Rolle bei der Pathogenese der AD spielen. Eine beachtliche Anzahl der in genetischen Studien identifizierten Risikogene weisen immunsystembezogene Funktionen auf und werden in höchstem Maße von Mikroglia exprimiert. Durch die Beschreibung von Risikovarianten in $C R 1$, CLU, SPI1, CD33, MS4A, ABCA7, EPHA1, HLADRB5/1, INPP5D, TYROBP, TREM2, PLCG2 und $A B / 3$ nimmt die Mikroglia vermittelte Immunantwort bei der Pathogenese der AD eine zentrale Rolle ein. Von besonderer Bedeutung könnte sein, dass die PLC $\gamma 2$ Variante p.P522R einen protektiven Effekt auf die LOAD (,llate-onset“ AD; spätmanifeste Form der $A D$ ) ausübt und als Enzym ein klassisches Ziel für eine therapeutische Modulation von komplexen Formen der AD darstellt.

Schlüsselwörter GWAS · Mutation · Risikofaktoren · Mikroglia · Immunantwort

\section{Genetics of Alzheimer's disease}

\section{Abstract}

Alzheimer's disease (AD) is the most common cause of neurodegenerative dementia. In contrast to monogenic and early-manifesting forms of $A D$ resulting from highly penetrant mutations in APP, PSEN1, and PSEN2, susceptibility to the sporadic, often lateonset $A D$ (LOAD) is determined by a complex interaction among genetic, epigenetic, and environmental-lifestyle-related factors. Although $A P O E \& 4$ is the strongest genetic risk factor for $A D$, its effect only accounts for $\sim 27.3 \%$ of the estimated disease heritability of $58-79 \%$. Through the continuing technical development of genome-wide association studies (GWAS) and next-generation automatic sequencing methods, scientists in large-scale collaborations have succeeded in gradually revealing the missing heritability. Important insights from GWAS and analyses of signaling pathways suggest that microglia, the resident immune cells of the CNS, play a decisive role in the pathogenesis of AD. A considerable number of risk genes identified in genetic studies indicate immune systemrelated functions and are expressed to a large extent by microglia. Through the description of risk variants in $C R 1, C L U, S P 11, C D 33, M S 4 A$, ABCA7, EPHA1, HLA-DRB5/1, INPP5D, TYROBP, TREM2, PLCG2, and $A B / 3$ the microgliamediated immune response takes on a central role in the pathogenesis of AD. Of note might be that the PLC 22 variant, p.P522R, exerts a protective effect on LOAD and as an enzyme, PLC $\gamma 2$ offers a classic target for the therapeutic modulation of complex forms of AD.

\section{Keywords} GWAS · Mutation · Risk factors · Microglia . Immune response 
ziationsmaß des APOE $\varepsilon 4$-Allels nah [2, $17,26]$. Eine zusätzliche Genvariante in TREM2, rs143332484 (p.R62H), wurde ebenfalls mit der LOAD in Verbindung gebracht sowie eine Reihe von weiteren SNPs, darunter rs142232675 (p.D87N) und rs2234253 (p.T96K) [17, 23, 56]. $\mathrm{Da}$ die o.g. kodierenden TREM2-Genvarianten äußerst selten sind, ist eine Bestimmung des LD erschwert. Dies liegt u.a. daran, dass eine allelische Assoziation zwischen den selteneren Genvarianten untereinander sowie zwischen diesen selteneren Genvarianten und aus bekannten häufigeren Varianten bestehenden LD-Blöcken innerhalb des TREM2-Locus noch nicht im Detail bekannt ist. Dass TREM2 jedoch ein Risikogen für die $\mathrm{AD}$ ist, wird durch funktionelle Untersuchungen untermauert [66]. Bei TREM2 handelt es sich um ein Immunrezeptor kodierendes Gen auf Chromosom 6p21.1 [30]. Im Vergleich $\mathrm{zu}$ Nichtträgern von TREM2 p.R47H zeigen AD-Patienten mit dieser Mutation früher einsetzende Symptome der Erkrankung [17], einen ausgeprägteren Hirnvolumenverlust [51] sowie Verluste an grauer Hirnsubstanz in betroffenen Regionen [38]. Molekulare Studien zeigen einen partiellen Funktionsverlust, was sich in einer vielseitig beeinträchtigten Mikrogliaaktivität äußert [66]. TREM2 konnte mit dem Lipidmetabolismus und insbesondere mit ApoE und ApoJ verknüpft werden, mit denen es direkt interagiert. Untersuchungen haben gezeigt, dass die in der extrazellulären Domäne lokalisierte p.R47H-Mutation eine beeinträchtigte Ligandenbindungskapazität bedingt und somit auch zu einer Veränderung der Lipidhomöostase führen könnte [67]. Neben der AD ist eine weitere Erkrankung mit genomischen Mutationen in TREM2 und TYROBP assoziiert, die Nasu-HakolaKrankheit, welche in Finnland und Japan endemisch ist. Es handelt sich um eine ausgesprochen seltene, autosomalrezessiv vererbte Erkrankung, die sich in einer fortschreitenden Demenz sowie einer polyzystischen Osteodysplasie äußert - Phänotypen, die sich beide mit dysfunktionalen TREM2/TYROBP-exprimierenden Zelltypen, Mikroglia und Osteoklasten, in Verbindung bringen las- sen [47]. Die Tatsache, dass TREM2 mit verschiedenen Demenzformen assoziiert ist, weist auf eine Beteiligung des Rezeptors an gemeinsamen neurodegenerativen Mechanismen und Signalwegen hin.

\section{PLCG2}

Im Jahr 2017 hat das IGAP-Konsortium eine seltene Variante in dem Gen PLCG2 (rs72824905, p.P522R, OR = 0,68; $\left.p=5,38 \times 10^{-10}\right)$ identifiziert, die sich protektiv auf die LOAD auszuwirken scheint und das Erkrankungsrisiko mindert [56]. PLCG2 kodiert für das Enzym PLC $\gamma 2$, welches das Membranphospholipid PIP $_{2}$ (Phosphatidylinositol-4,5-bisphosphat) $\mathrm{zu}$ den intrazellulären Mediatoren $\mathrm{IP}_{3}$ (Inositol-1,4,5-trisphosphat) und DAG (Diacylglycerol) hydrolysiert [36]. Während der $\mathrm{IP}_{3}$-DAG-Ca ${ }^{2+}$-Signalweg in diversen Zelltypen aktiv ist, beschränkt sich die Expression von PLCG2 im Gehirngewebe hauptsächlich auf mikrogliale Zellen [68]. Dass PLC 22 p.P522R einen protektiven Effekt auf die LOAD ausübt und als Enzym ein klassisches Ziel für eine therapeutische Modulation bietet, könnte von besonderer Bedeutung sein. Eine mögliche Rolle von PLC $\gamma 2$ bei immunvermittelten Prozessen an der AD wird durch die Beschreibung von kleinen heterozygoten Deletionen im kodierenden Bereich und „missense“ Mutationen unterstrichen, die für autosomal-dominant vererbte immundysregulatorische Defekte wie PLAID („PLC $\gamma 2$-associated antibody deficiency and immune dysregulation") und APLAID (,autoinflammation and PLAID“) verantwortlich sind $[14,46,69]$. Die Mechanismen, wie PLAID/APLAID-assoziierte Mutationen zur jeweiligen Erkrankung führen, sind hochkomplex und äußern sich in einem Funktionsgewinn wie auch Funktionsverlust von PLC $\gamma 2$ in zellulären Signalwegen. Häufig kommt es durch die Mutationen zu einer Beeinträchtigung der cSH2 (,carboxy terminal Src-homology 2“) autoinhibitorischen Domäne, was zu einer kompromittierten Aktivität des Enzyms führen kann [43, 65]. Darüber hinaus wurden PLCG2Mutationen mit der Ibrutinib-resistenten chronischen lymphatischen Leukämie in
Verbindung gebracht [64]. Wie sich die AD-assoziierte p.P522R-Mutation, die außerhalb der SH2-Domänen lokalisiert ist, auf PLC $\gamma 2$ und PLC $\gamma 2$-vermittelte Prozesse auswirkt, ist zurzeit Gegenstand intensiver Forschung.

$A B I 3$

Die Genvariante p.S209F (rs616338, $\mathrm{OR}=1,43 ; p=4,56 \times 10^{-10}$ ) in $A B I 3$ wurde vor Kurzem als Risikofaktor für die LOAD identifiziert [56]. Wie auch die Genprodukte von PLCG2 und TREM2 nimmt ABI3 (über Interferon-gesteuerte Signaltransduktion) eine immunsystembezogene Funktion wahr [12]. Zusammen mit INPP5D, das bereits mit dem LOAD-Risiko in Zusammenhang gebracht wurde [34], wird $A B I 3$ co-exprimiert. Weiterhin spielt es eine wichtige Rolle bei der Organisation des Aktinzytoskeletts über den WAVE2Komplex („Wiskott-Aldrich syndrome protein family verprolin-homologous protein 2“) [55]. Dieser Komplex reguliert unter anderem Signalwege, die zur Aktivierung von T-Zellen führen [45].

\section{Interaktion von immunsystem- bezogenen Proteinen bei der LOAD}

Wichtige Erkenntnisse aus der Genforschung weisen auf die Beteiligung von Mikroglia vermittelten Prozessen bei der Pathogenese der AD hin. Gene, welche die Suszeptibilität für die AD modifizieren, lassen sich insbesondere der angeborenen Immunantwort zuordnen [19]. Ein von Sims und Kollegen konstruiertes, aus 56 Genen bestehendes Interaktionsnetzwerk, beinhaltet eine Vielzahl von mikroglialen, immunassoziierten Genen, darunter TREM2, PLCG2, ABI3, SPI1, INPPD5 und TYROBP [56]. Als myeloider Schlüsseltranskriptionsfaktor übernimmt das Genprodukt von SPI1, PU.1, eine zentrale Rolle bei der homöostatischen Funktion von Mikroglia [58]. Die Tyrosinkinase Syk, welche indirekt mit dem TREM2/DAP12-Komplex assoziiert ist, vermittelt unter anderem die Phosphorylierung von PLC $\gamma 2$ [32] und moduliert die Produktion von $A \beta$ und die Tau-Hyperphosphorylierung [48]. 
Hier steht eine Anzeige.

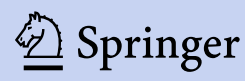


Über den INPP5D-CD2AP-Komplex wird die Degradation von Syk reguliert [1]. Darüber hinaus wird der blutdrucksenkende $\mathrm{Ca}^{2+}$-Antagonist, Nilvadipin, welcher inhibierend auf Syk wirkt, in einer klinischen Phase-III-AD-Studie begutachtet; das Ergebnis bleibt abzuwarten ([35], Clinical Trial Identifier: NCT02017340). Neben SPI1 und SYK sind auch TREM2, TYROBP und PLCG2 zentrale, miteinander verbundene Knotenpunkte in einem mikroglialen, immunassoziierten Gennetzwerk [41, 50, 56]. Durch die Beteiligung von ABI 3 an der Regulation des Aktinzytoskeletts [55] wäre es denkbar, dass sich eine Fehlfunktion von ABI3 auf TREM2-vermittelte, chemotaktische und phagozytotische Prozesse auswirken könnte [29, 42]. Die Identifikation dieser LOAD-assoziierten Gene und funktionelle Untersuchungen erbringen eindeutige Hinweise dafür, dass eine mikrogliale Dysfunktion eine ursächliche Rolle bei der LOAD spielen könnte. Zukünftige Studien werden aufklären, ob Mutationen in diesen verschiedenen Genen zur Beeinträchtigung von gemeinsamen Signalwegen führen und wie die Genprodukte zur therapeutischen Modulation der LOAD genutzt werden könnten.

\section{Klinische Anwendungsaspekte}

Ein weiteres Ziel der Genforschung ist es, Ansätze zu entwickeln, um Befunde routinemäßig in die klinische Praxis einzubringen. Die Charakterisierung von hochpenetranten Mutationen in $A P P$, PSEN1 und PSEN2 erbrachte wertvolle Erkenntnisse, die Anwendung in Diagnose und Arzneimittelentwicklung finden. Mittels spezifischer Gentests können potenziell von EOAD betroffene Familienmitglieder auf die Vererbung von Genmutationen untersucht werden. Weil die $\mathrm{AD}$ nicht heilbar ist und zurzeit keine besonders wirksame Therapie zur Linderung der Alzheimer-Symptome bzw. zur Veränderung des Krankheitsverlaufs existiert, nimmt in diesem Zusammenhang ein multidisziplinäres Team aus Klinikern, Genetikern und Psychologen eine entscheidende Rolle bei der Mitteilung eines positiven Befundes ein [16]. Mutationen in den o.g. Genen sind al- lerdings äußerst selten und finden sich nur in $5-10 \%$ der Fälle [5]. Dies lässt einen großen Teil der Fälle ungeklärt und impliziert auch, dass weitere, noch unidentifizierte Gene der EOAD zugrunde liegen könnten. In diesem Zusammenhang spielen die rasanten Entwicklungen von neuen Technologien (Sequenziermethoden der nächsten Generation) in der Genforschung eine wichtige Rolle für die Aufdeckung von zusätzlichen Genen und pathogenen Mutationen. Diese neuen Erkenntnisse erweitern kontinuierlich die Möglichkeiten der Molekulardiagnostik und erhöhen somit die Wahrscheinlichkeit einer molekularen Aufklärung von bisher ungelösten Fällen. Die Anwendung von Befunden aus GWAS in der klinischen Praxis wird allerdings durch zwei Gründe erschwert: Die identifizierten Signale stellen womöglich nicht die ursächlichen Varianten dar und üben nur eine niedrige bis moderate Wirkung auf das Krankheitsrisiko aus [10]. Sogar das APOE \&4-Allel, welches die höchste OR aufweist, ist allein für eine Krankheitsmanifestation nicht ausreichend. Allerdings beträgt das in einer Studie von Genin und Kollegen errechnete Lebenszeitrisiko (LZR) an AD zu erkranken für weibliche Individuen im Alter von 85 Jahren mit dem APOE $\varepsilon 4 / \varepsilon 4$-Genotyp $60 \%$. Bei männlichen Trägern ist das LZR etwas geringer und beläuft sich auf $51 \%$. Im Vergleich dazu ist das LZR für heterozygo-

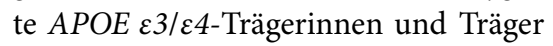
deutlich herabgesenkt (30\% bzw. $23 \%$ ). Ungeachtet des APOE-Genotyps beläuft sich das LZR in dieser Studie auf $11 \%$ (männl. Individuen) und 14\% (weibl. Individuen) [15]. Folglich stellt sich die Frage, ob im Rahmen humangenetischer Beratung und Diagnostik der APOE-Genotyp bestimmt werden sollte. Diesbezüglich hat die Forschung zeigen können, dass eine präventive Intervention durch Veränderungen in der Lebensweise den genetisch bedingten Effekten des APOE $\varepsilon 4$ entgegenwirkt [13]. Mit dem Vorhaben, die in GWAS identifizierten Signale effektiver nutzen zu können, wird vermehrt auf die Bildung eines genetischen Risk-Scores („genetic risk score“, GRS) hingearbeitet, welcher auf kumulativen Effekten aus individuellen Suszeptibilitätsvarianten basiert. Obwohl die Signale aus GWAS und die Ergebnisse von GRSTests für eine Prädiktion oder klinische Diagnose der AD nicht eindeutig sind, liegt ihr großer Nutzen darin, frühzeitig Individuen zu identifizieren, die ein gesteigertes AD-Risiko haben. Eingeschlossen in therapeutische oder BiomarkerStudien, könnten diese AD-Risikopersonen zu eindeutigeren Ergebnissen beitragen [57]. Weiterhin ist in naher $\mathrm{Zu}$ kunft eine medikamentöse Heilung oder Verzögerung des Krankheitsverlaufs der $\mathrm{AD}$ nicht zu erwarten; daher kann bei frühzeitiger Identifizierung von AD-Risikopersonen ein verstärkter Fokus auf der Krankheitsprävention liegen [9].

\section{Fazit für die Praxis}

\section{- Obgleich die durch die Genforschung gewonnenen Erkenntnisse noch kei- ne umfassende klinische Anwendung gefunden haben, unterstreichen sie ihren Nutzen für die Erstellung eines genetischen "risk score", der eine frühzeitige Identifikation von Individuen mit erhöhtem AD-Risiko ermöglicht. Diesbezüglich hat die Forschung gezeigt, dass präventive Maßnahmen genetischen Risikofak- toren, wie beispielsweise dem APOE $\varepsilon 4-A l l e l$, entgegenwirken können. \\ - Wichtige Erkenntnisse aus GWAS und Signalweganalysen suggerieren, dass Mikroglia eine entscheidende Rolle bei der Pathogenese der AD spielen. Vor allem die Identifikation von PLCY2 p.P522R eröffnet eine vielversprechende Möglichkeit für eine therapeutische Modulation der AD.}

\section{Korrespondenzadresse}

\section{PD Dr. Dr. A. Ramirez}

Sektion für Neurogenetik und Molekulare Neuropsychiatrie an der Klinik für Psychiatrie und Psychotherapie, Uniklinik Köln Kerpener Straße 62, 50937 Köln, Deutschland alfredo.ramirez@uk-koeln.de alfredo.ramirez@ukbonn.de 


\section{Einhaltung ethischer Richtlinien}

Interessenkonflikt. T. Bajaj, A. Ramirez und H. Wag ner-Thelen geben an, dass kein Interessenkonflikt besteht.

Dieser Beitrag beinhaltet keine von den Autoren durchgeführten Studien an Menschen oder Tieren.

Open Access Dieser Artikel wird unter der Creative Commons Namensnennung 4.0 International Lizenz (http://creativecommons.org/licenses/by/4.0/deed. de) veröffentlicht, welche die Nutzung, Vervielfältigung, Bearbeitung, Verbreitung und Wiedergabe in jeglichem Medium und Format erlaubt, sofern Sie den/die ursprünglichen Autor(en) und die Quelle ordnungsgemäß nennen, einen Linkzur Creative Commons Lizenz beifügen und angeben, ob Änderungen vorgenommen wurden.

\section{Literatur}

1. Bao M, Hanabuchi S, Facchinetti V et al (2012) CD2AP/SHIP1 complex positively regulates plasmacytoid dendritic cell receptor signaling by inhibiting the $\mathrm{E} 3$ ubiquitin ligase $\mathrm{Cbl}$. J Immunol 189:786-792

2. Bertram L, Mcqueen MB, Mullin K et al (2007) Systematic meta-analyses of Alzheimer disease genetic association studies: the AlzGene database. Nat Genet 39:17-23

3. Bradshaw EM, Chibnik LB, Keenan BT et al (2013) CD33 Alzheimer's disease locus: altered monocyte function and amyloid biology. Nat Neurosci 16:848-850

4. Brouwers N, Van Cauwenberghe C, Engelborghs $S$ et al (2012) Alzheimer risk associated with a copy number variation in the complement receptor 1 increasing $\mathrm{C} 3 \mathrm{~b} / \mathrm{C} 4 \mathrm{~b}$ binding sites. Mol Psychiatry 17:223-233

5. Cacace R, Sleegers K, Van Broeckhoven C (2016) Molecular genetics of early-onset Alzheimer's disease revisited. Alzheimers Dement 12:733-748

6. Chibnik LB, Shulman JM, Leurgans SE et al (2011) CR1 is associated with amyloid plaque burden and age-related cognitive decline. Ann Neurol 69:560-569

7. Conejero-Goldberg C, Gomar JJ, Bobes-Bascaran T et al (2014) APOE2 enhances neuroprotection against Alzheimer's disease through multiple molecular mechanisms. Mol Psychiatry 19:1243-1250

8. Corder EH, Saunders AM, Strittmatter WJ et al (1993) Gene dose of apolipoprotein E type 4 allele and the risk of Alzheimer's disease in late onset families. Science 261:921-923

9. Escott-Price V, Sims R, Bannister Cet al (2015) Common polygenic variation enhances risk prediction for Alzheimer's disease. Brain 138:3673-3684

10. Evangelou E, loannidis JP (2013) Meta-analysis methods forgenome-wide association studies and beyond. Nat Rev Genet 14:379-389

11. Evans RM, Hui S, Perkins A et al (2004) Cholesterol and APOE genotype interact to influence Alzheimer disease progression. Neurology 62:1869-1871

12. Fairfax BP, Humburg $P$, Makino S et al (2014) Innate immune activity conditions the effect of regulatory variants upon monocyte gene expression. Science 343:1246949

13. Fyfe I (2018) Alzheimer disease: APOE epsilon4 affects cognitive decline but does not block benefits of healthy lifestyle. Nat Rev Neurol 14:125
14. Gandhi C, Healy C, Wanderer AA, Hoffman HM (2009) Familial atypical cold urticaria: description of a new hereditary disease. J Allergy Clin Immunol 124:1245-1250

15. Genin E, Hannequin D, Wallon D et al (2011) APOE and Alzheimer disease: a major gene with semidominant inheritance. Mol Psychiatry 16:903-907

16. Goldman JS, Hahn SE, Catania JW et al (2011) Genetic counseling and testing for Alzheimer disease: joint practice guidelines of the American College of Medical Genetics and the National Society of Genetic Counselors. Genet Med 13:597-605

17. Guerreiro R, Wojtas A, Bras J et al (2013) TREM2 variants in Alzheimer's disease. N Engl J Med 368:117-127

18. Harold D, Abraham R, Hollingworth P et al (2009) Genome-wide association study identifies variants at CLU and PICALM associated with Alzheimer's disease. Nat Genet 41:1088-1093

19. Heneka MT, Carson MJ, El Khoury J et al (2015) Neuroinflammation in Alzheimer's disease. Lancet Neurol 14:388-405

20. Holers VM (2014) Complement and its receptors: new insights into human disease. Annu Rev Immunol 32:433-459

21. Hollingworth $P$, Harold D, Sims R et al (2011) Common variants at ABCA7, MS4A6A/MS4A4E, EPHA1, CD33 and CD2AP are associated with Alzheimer's disease. Nat Genet 43:429-435

22. Huang YA, Zhou B, Wernig $M$ et al (2017) ApoE2, ApoE3, and ApoE4 differentially stimulate APP transcription and Abeta secretion. Cell 168:427-441.e21

23. Jin SC, Benitez BA, Karch CM et al (2014) Coding variants in TREM2 increase risk for Alzheimer's disease. Hum Mol Genet 23:5838-5846

24. Jones L, Holmans PA, Hamshere ML et al (2010) Genetic evidence implicates the immune system and cholesterol metabolism in the aetiology of Alzheimer's disease. PLoS ONE 5:e13950

25. Jonsson T, Atwal JK, Steinberg S et al (2012) A mutation in APP protects against Alzheimer's disease and age-related cognitive decline. Nature 488:96-99

26. Jonsson T, Stefansson H, Steinberg S et al (2013) Variant of TREM2 associated with the risk of Alzheimer's disease. NEngl J Med 368:107-116

27. Kanekiyo T, Xu H, Bu G (2014) ApoE and Abeta in Alzheimer's disease: accidental encounters or partners? Neuron 81:740-754

28. Karch CM, Jeng AT, Nowotny P et al (2012) Expression of novel Alzheimer's disease risk genes in control and Alzheimer's disease brains. PLoS ONE 7:e50976

29. Kleinberger G, Yamanishi Y, Suarez-Calvet $M$ et al (2014) TREM2 mutations implicated in neurodegeneration impair cell surface transport and phagocytosis. Sci Transl Med 6:243ra286

30. Klesney-Tait J, Turnbull IR, Colonna M (2006) The TREM receptor family and signal integration. Nat Immunol 7:1266-1273

31. Krawczak M (2014) Genomweite Assoziationsstudien (GWAS). In: Lenk C, Duttge G, Fangerau H (Hrsg) Handbuch Ethik und Recht der Forschung am Menschen. Springer, Berlin, Heidelberg, S39-42

32. Kurosaki T, Tsukada S (2000) BLNK: connecting Syk and Btk to calcium signals. Immunity 12:1-5

33. Lambert JC, Heath S, Even G et al (2009) Genomewide association study identifies variants at CLU and CR1 associated with Alzheimer's disease. Nat Genet 41:1094-1099
34. Lambert JC, Ibrahim-Verbaas CA, Harold D et al (2013) Meta-analysis of 74,046 individuals identifies 11 new susceptibility loci for Alzheimer's disease. Nat Genet 45:1452-1458

35. Lawlor B, Kennelly S, O'dwyer S et al (2014) NILVAD protocol: a European multicentre double-blind placebo-controlled trial of nilvadipine in mild-tomoderate Alzheimer's disease. BMJOpen 4:e6364

36. Lee SB, Rhee SG (1995) Significance of PIP2 hydrolysis and regulation of phospholipase $C$ isozymes. Curr Opin Cell Biol 7:183-189

37. Levy-Lahad E, Wasco W, Poorkaj P et al (1995) Candidate gene for the chromosome 1 familial Alzheimer's disease locus. Science 269:973-977

38. Luis EO, Ortega-Cubero S, Lamet I et al (2014) Frontobasal gray matter loss is associated with the TREM2 p.R47H variant. Neurobiol Aging 35:2681-2690

39. Mahley RW, Weisgraber KH, Huang Y (2006) Apolipoprotein $\mathrm{E} 4$ : a causative factor and therapeutic target in neuropathology, including Alzheimer's disease. Proc Natl Acad Sci USA 103:5644-5651

40. Malik M, Simpson JF, Parikh I et al (2013) CD33 Alzheimer's risk-altering polymorphism, CD33 expression, and exon 2 splicing. J Neurosci 33:13320-13325

41. Mao D, Epple H, Uthgenannt B et al (2006) PLCgamma2 regulates osteoclastogenesis via its interaction with ITAM proteins and GAB2. J Clin Invest 116:2869-2879

42. Mazaheri F, Snaidero N, Kleinberger $G$ et al (2017) TREM2 deficiency impairs chemotaxis and microglial responses to neuronal injury. Embo Rep 18:1186-1198

43. Milner JD (2015) PLAID: a syndrome of complex patterns of disease and unique phenotypes. J Clin Immunol 35:527-530

44. Naj AC, Jun G, Beecham GW et al (2011) Common variants at MS4A4/MS4A6E, CD2AP, CD33 and EPHA1 are associated with late-onset Alzheimer's disease. Nat Genet 43:436-441

45. Nolz JC, Gomez TS, Zhu P et al (2006) The WAVE2 complex regulates actin cytoskeletal reorganization and CRAC-mediated calcium entry during T cell activation. Curr Biol 16:24-34

46. Ombrello MJ, Remmers EF, Sun G et al (2012) Cold urticaria, immunodeficiency, and autoimmunity related to PLCG2 deletions. $\mathrm{N}$ Engl J Med 366:330-338

47. Paloneva J, Manninen T, Christman G et al (2002) Mutations in two genes encoding different subunits of a receptor signaling complex result in an identical disease phenotype. Am J Hum Genet 71:656-662

48. Paris D, Ait-Ghezala G, Bachmeier C et al (2014) The spleen tyrosine kinase (Syk) regulates Alzheimer amyloid-beta production and Tau hyperphosphorylation. JBiol Chem 289:33927-33944

49. Peiper SC, Ashmun RA, Look AT (1988) Molecular cloning, expression, and chromosomal localization of a human gene encoding the CD33 myeloid differentiation antigen. Blood 72:314-321

50. Pottier C, Ravenscroft TA, Brown PH et al (2016) TYROBPgenetic variants in early-onset Alzheimer's disease. Neurobiol Aging 48:222.e9-222.e15

51. Rajagopalan P, Hibar DP, Thompson PM (2013) TREM2 and neurodegenerative disease. N Engl J Med 369:1565-1567

52. Ridge PG, Mukherjee S, Crane PK et al (2013) Alzheimer's disease: analyzing the missing heritability. PLoSONE 8:e79771

53. Ruiz A, Heilmann S, Becker T et al (2014) Followup of loci from the International Genomics of Alzheimer's Disease Project identifies TRIP4 as 
a novel susceptibility gene. Transl Psychiatry 4:e358

54. Saunders AM, Strittmatter WJ, Schmechel D et al (1993) Association of apolipoprotein E allele epsilon 4 with late-onset familial and sporadic Alzheimer's disease. Neurology 43:1467-1472

55. Sekino S, Kashiwagi Y, Kanazawa H et al (2015) The NESH/Abi-3-based WAVE2 complex is functionally distinct from the Abi-1-based WAVE2 complex. Cell Commun Signal 13:41

56. Sims R, Van Der Lee SJ, Naj AC et al (2017) Rare coding variants in PLCG2, ABI3, and TREM2 implicate microglial-mediated innate immunity in Alzheimer's disease. Nat Genet 49:1373-1384

57. Sleegers K, Bettens K, De Roeck A et al (2015) A 22-single nucleotide polymorphism Alzheimer's disease risk score correlates with family history, onset age, and cerebrospinal fluid Abeta42. Alzheimers Dement 11:1452-1460

58. Smith AM, Gibbons HM, Oldfield RL et al (2013) The transcription factor PU. 1 is critical for viability and function of human brain microglia. Glia 61:929-942

59. St George-Hyslop PH, Tanzi RE, Polinsky RJ et al (1987) The genetic defect causing familial Alzheimer's disease maps on chromosome 21. Science 235:885-890

60. Stampfer MJ (2006) Cardiovascular disease and Alzheimer's disease: common links. J Intern Med 260:211-223

61. Van Broeckhoven C, Backhovens $H$, Cruts $M$ et al (1992) Mapping of a gene predisposing to early-onset Alzheimer's disease to chromosome 14q24.3. Nat Genet 2:335-339

62. Van Cauwenberghe C, Van Broeckhoven C, Sleegers K (2016) The genetic landscape of Alzheimer disease: clinical implications and perspectives. Genet Med 18:421-430

63. Van Duijn CM, De Knijff P, Cruts M et al (1994) Apolipoprotein E4 allele in a population-based study of early-onset Alzheimer's disease. Nat Genet 7:74-78

64. Walliser C, Hermkes E, Schade A et al (2016) The phospholipase Cgamma2 mutants R665W and L845F identified in Ibrutinib-resistant chronic lymphocytic leukemia patients are hypersensitive to the rho GTPase Rac2 protein. J Biol Chem 291:22136-22148

65. Wang J, Sohn H, Sun G et al (2014) The autoinhibitory C-terminal $\mathrm{SH} 2$ domain of phospholipase $\mathrm{C}-{ }^{2} 2$ stabilizes B cell receptor signalosome assembly. Sci Signal 7(343):ra89. https://doi.org/10.1126/ scisignal.2005392

66. Yeh FL, Hansen DV, Sheng M (2017) TREM2, microglia, and neurodegenerative diseases. Trends Mol Med 23:512-533

67. Yeh FL, Wang Y, Tom l et al (2016) TREM2 binds to apolipoproteins, including APOE and CLU/APOJ, and thereby facilitates uptake of amyloid-beta by microglia. Neuron 91:328-340

68. Zhang Y, Sloan SA, Clarke LE et al (2016) Purification and characterization of progenitor and mature human astrocytes reveals transcriptional and functional differences with mouse. Neuron 89:37-53

69. Zhou Q, Lee GS, Brady J et al (2012) A hypermorphic missense mutation in PLCG2, encoding phospholipase $\mathrm{C} \gamma 2$, causes a dominantly inherited autoinflammatory disease with immunodeficiency. Am JHum Genet 91:713-720

\section{Erstmals Proteom des gesunden menschlichen Herzens entschlüsselt}

Ein gesundes Herz schlägt etwa zwei Milliarden Mal im Leben. Dafür sorgen mehr als 10.000 Proteine. Welche und wie viele einzelne Proteine in welchen Zelltypen vorhanden sind, haben jetzt Forscher des Max-Planck-Instituts für Biochemie (MPIB) und des Deutschen Herzzentrums München an der Technischen Universität München (TUM) erfasst. Sie haben den ersten Herzatlas des gesunden menschlichen Herzens erstellt. Damit lassen sich in Zukunft Unterschiede zwischen kranken und gesunden Herzen aufdecken.

Entstehen auf DNA- oder Protein-Ebene Veränderungen, können Krankheiten entstehen. Damit solche Veränderungen als Ursachen für Herzkrankheiten erkannt werden können, ist es wichtig zu wissen, wo welche Proteine im gesunden Herzen vorhanden sind und in welcher Menge sie vorliegen.

\section{Proteinatlas des Herzens}

Das Proteom für das gesmte Herz konnte ein Forscherteam aus München jetzt in „Nature Communications" veröffentlichen. Dafür bestimmten die Wissenschaftler die komplette Proteinausstattung der Zellen in allen Regionen des Herzens wie Herzklappen, Herzkammern und den wichtigsten Blutgefäßen. Zudem untersuchten sie die Proteinzusammensetzung in drei unterschiedlichen Zelltypen des Herzens: den Herzfibroblasten, den glatten Muskelzellen und den Endothelzellen. So konnten sie die Verteilung der Proteine in den unterschiedlichen Herzbereichen darstellen. Mit Hilfe der Massenspektrometrie konnten fast 11.000 unterschiedliche Proteine im gesamten Herz identifiziert werden.

Das Ergebnis zeigt: Alle gesunden Herzen funktionieren sehr ähnlich. Die Wissenschaftler konnten in den einzelnen Regionen jeweils eine ähnliche Proteinzusammensetzung messen, die nur wenige individuelle Unterschiede zeigte. Überraschend war auch, dass die rechte und linke Herzhälfte sich glichen, obwohl sie unterschiedliche Aufgaben übernehmen.

\section{Krank vs. Gesund: Individuelle}

\section{Unterschiede erkennen}

Im nächsten Schritt wollten die Autoren testen, ob sich mit den Daten der gesunden Herzen als Kontrolle auch Veränderungen in kranken Herzen erkennen lassen. Sie verglichen ihre Werte mit Herzproteomen von Patienten mit Vorhofflimmern. Die Ergebnisse konnten tatsächlich erste Hinweise auf die Ursache der Krankheit liefern: Das Gewebe des kranken Herzens unterschied sich am stärksten bei Proteinen, die für die Energieversorgung der Zelle verantwortlich waren.

Der Vergleich lieferte noch ein weiteres interessantes Ergebnis: Zwar waren bei allen Patienten die Proteine des Energiestoffwechsels verändert, aber bei jedem gab es individuelle Veränderungen. Diese Ergebnisse zeigen, wie wichtig die personalisierte Medizin ist. Obwohl alle Patienten sehr ähnliche Symptome haben, sahen die Forscher, dass bei allen Patienten eine unterschiedliche molekulare Fehlfunktion zugrunde liegt. In Zukunft muss die Herzmedizin lernen, solche individuellen Unterschiede zu erkennen und zu behandeln.

Literatur: Doll S, DreßenM , Geyer PE et al (2017) Region and cell-type resolved quantitative proteomic map of the human heart, $\mathrm{Na}$ ture Communications, DOI: 10.1038/s41467017-01747-2

Quelle: Technische Universität München 